\title{
Comparative transcriptomic analysis between in vivo derived porcine 4-cell and morula embryos
}

\author{
C. Zhou, S. Tsoi, W.T. Dixon, G.R. Foxcroft and M.K. Dyck \\ Swine Reproduction-Development Program, Swine Research \& Technology Centre, University of \\ Alberta, Edmonton, Alberta, CANADA T6G 2P5
}

During the pre-implantation period of embryonic development, the porcine embryo exhibits dramatic morphological changes associated with key developmental events such as embryonic genome activation (EGA). In mammals, oocyte-derived mRNAs are degraded shortly after fertilization, hence embryonic genome activation and production of embryo-derived transcripts must occur during early embryonic development (Thompson, Legouy \& Renard 1998, Schultz 2002). EGA is a gradual process: a small portion of the embryo genome activates early and the major embryonic genome activation of abundant transcription occur later (Oestrup et al. 2009). The molecular mechanisms underlying these events are not yet fully understood. To better elucidate these mechanisms at the gene expression level, a comparative transcriptomic analysis between in vivo-derived 4-cell and morula stage porcine embryos was performed with with a custom designed porcine embryo-specific microarray platform (EMPV1: EmbryoGENE Porcine Array Version1 [GPL14925]) (Tsoi et al. 2012) (http://embryogene.ca/). In this study, the microarray analysis was performed following a reference design (Novoradovskaya et al. 2004, Konig et al. 2004) using a reference RNA pool generated from 10 different porcine embryonic stages (GV, MII, 2-cell, 4-cell, 8-cell, morula, early blastocyst, expanded blastocyst, hatched blastocyst stages and day 11 embryos).

In-vivo derived porcine embryos from 4-cell and morula stages were collected from gilts as described previously (Degenstein et al. 2008). All embryo samples were placed on dry ice immediately after collection and stored at $-80^{\circ} \mathrm{C}$. Total RNA samples were extracted from pools of five embryos using Arcturus ${ }^{\circledR}$ PicoPure ${ }^{\circledR}$ RNA Isolation Kit (Applied Biosystems, CA, USA). Total RNA samples were amplified using RiboAmp HS Plus kit (Applied Biosystems, CA, USA) following manufacturer's instructions and generated amplified antisense RNA (aRNA) targets for microarray reactions. Each aRNA sample was labelled with Cy5 dye and hybridized with Cy3 dye labelled reference RNA pool on EMPV1 Microarray. Three biological replicates from each group were included in the comparative microarray analysis. Agilent two-colour RNA Spike-In ${ }^{\circledR}$ (Agilent Technologies, Mississauga, ON, Canada) were amplified, labelled and utilized as positive control in each hybridization as previously described (Tsoi et al. 2012). Microarray data analysis was performed using FlexArray software 1.6.1 (http://genomequebec.mcgill.ca/ FlexArray). Simple background subtraction and within array lowess global normalization was performed on raw data from each array. The limma software (Smyth 2005) was utilized to identify the differentially expressed genes between different stages. Ingenuity ${ }^{\circledR}$ Pathway analysis (IPA) software were used for the biological process and pathway analysis. IPA Upstream Regulator Analysis tool was used in transcription factor identification and activation status (activated or inhibited) prediction using the regulation z-score algorithm ( $|z| \geq 2$ is considered significant), which is calculated based on the direction of expression changes (up-regulate or down-regulate) of known transcriptional regulation targets in the experimental dataset and the direction of expectations derived from literature.

The comparative analysis revealed 2,582 differentially expressed genes between 4-cell and morula stage embryos. A major portion of these differentially expressed genes are associated 
with "embryonic development" (153 genes), "RNA post-transcriptional modification" (78 genes), and "energy production" (60 genes). IPA canonical pathways analysis of all the differentially expressed genes showed that "EIF2 (eukaryotic translation initiation factor-2) signaling" (95 genes), "mTOR Signaling" (63 genes), as well as "regulation of elF4 (Eukaryotic translation initiation factor 4) and p70S6K Signaling" (65 genes), are the three most significant canonical pathways.

IPA Upstream Regulator analysis of all the differentially expressed genes between 4-cell and morula identified 95 regulation targets of the transcription factor MYCN (Avian neuroblastoma derived V-myc myelocytomatosis viral related oncogene). MYCN was predicted to be the most significantly activated transcription regulator from 4-cell to morula stage (Z score: 6.181, p-value: 3.71E-28). Microarray data shows that the transcription factor MYCN exhibited a 4.8 fold up-regulation from 4-cell to morula stage. Furthermore, IPA canonical pathway analysis of these 95 MYCN target genes showed that most of them are associated with the "EIF2 signaling" (47 genes), "mTOR Signaling" (19 genes), as well as the "regulation of elF4 and p70S6K signaling" (22 genes) pathways, which represents more than 1/3 of all differentially expressed genes associated with these 3 pathways. These three pathways play very important roles during the development of early porcine embryos. The "elF2 signaling pathway" and the "regulation of elF4 and p70S6K signaling" pathways play critical roles in the regulation of general protein synthesis and mRNA translation (Wek, Jiang \& Anthony 2006). The "mTOR signaling pathway" is the central regulator of cell metabolism, translation, growth, proliferation and survival (Laplante, Sabatini 2009).

In pig, the major wave of embryonic genome activation (EGA) occurs between 4-cell stage and morula stage (Oestrup et al. 2009). The results from this study suggest that the transcription factor MYCN is activated after 4-cell stage, and further regulated the expression of many genes associated with cell metabolism, protein synthesis, growth, proliferation and survival during EGA. Activation of transcription factor MYCN during early EGA probably is necessary for the successful development of early porcine embryo.

\section{References}

Degenstein, K.L., O’Donoghue, R., Patterson, J.L., Beltranena, E., Ambrose, D.J., Foxcroft, G.R. \& Dyck, M.K. 2008 Synchronization of ovulation in cyclic gilts with porcine luteinizing hormone $(\mathrm{pLH})$ and its effects on reproductive function. Theriogenology 70, no. 7, pp. 1075-1085.

Konig, R., Baldessari, D., Pollet, N., Niehrs, C. \& Eils, R. 2004 Reliability of gene expression ratios for CDNA microarrays in multiconditional experiments with a reference design. Nucleic acids research 32 no. 3, pp. e29.

Laplante, M. \& Sabatini, D.M. 2009 mTOR signaling at a glance. Journal of cell science $\mathbf{1 2 2}$ no. Pt 20, pp. 35893594.

Novoradovskaya, N., Whitfield, M.L., Basehore, L.S., Novoradovsky, A., Pesich, R., Usary, J., Karaca, M., Wong, W.K., Aprelikova, O., Fero, M., Perou, C.M., Botstein, D. \& Braman, J. 2004 Universal Reference RNA as a standard for microarray experiments. $B M C$ genomics 5, no. 1, pp. 20.

Oestrup, O., Hall, V., Petkov, S.G., Wolf, X.A., Hyldig, S. \& Hyttel, P. 2009 From zygote to implantation: morphological and molecular dynamics during embryo development in the pig. Reproduction in domestic animals 44 Suppl 3, pp. 39-49.
Schultz, R.M. 2002 The molecular foundations of the maternal to zygotic transition in the preimplantation embryo, Human reproduction update. 8 no. 4, pp. 323-331.

Smyth, G.K. 2005 limma: Linear Models for Microarray Data in Bioinformatics and Computational Biology Solutions Using $R$ and Bioconductor, eds. R. Gentleman, V. Carey, W. Huber, R. Irizarry \& S. Dudoit, Springer New York, pp. 397-420.

Thompson, E.M., Legouy, E. \& Renard, J. 1998 Mouse embryos do not wait for the MBT: Chromatin and RNA polymerase remodeling in genome activation at the onset of development. Developmental genetics 22, no. 1, pp. 31-42.

Tsoi, S., Zhou, C., Grant, J., Pasternak, A., Dobrinsky, J., Rigault, P., Nieminen, J., Sirard, M., Robert, C., Foxcroft, G. \& Dyck, M. 2012 Development of a porcine (Sus scofa) embryo-specific microarray: array annotation and validation. BMC Genomics 13, no. 1, pp. 370.

Wek, R.C., Jiang, H.Y. \& Anthony, T.G. 2006 Coping with stress: elF2 kinases and translational control. Biochemical Society transactions 34, no. Pt 1, pp. 7-11. 